\title{
Phase Errors Simulation Analysis for GNSS Antenna in Multipath Environment
}

\author{
Lixun Li, Baiyu Li, Huaming Chen, and Feixue Wang \\ College of Electronic Science and Engineering, National University of Defense Technology, Changsha 410073, China \\ Correspondence should be addressed to Feixue Wang; wangfeixue_nnc@163.com
}

Received 6 September 2015; Accepted 15 October 2015

Academic Editor: Jaume Anguera

Copyright (C) 2015 Lixun Li et al. This is an open access article distributed under the Creative Commons Attribution License, which permits unrestricted use, distribution, and reproduction in any medium, provided the original work is properly cited.

\begin{abstract}
High-precision GNSS application requires the exact phase center calibration of antenna. Various methods are published to determine the locations of the phase center. In the outfield, when the phase errors that arose by multipath exceed the phase center variations (PCV) tolerance, the calibration values may be not useful. The objective of this paper is thus to evaluate the phase errors that arose by multipath signals. An improved model of antenna receiving signal is presented. The model consists of three main components: (1) an antenna model created by combination of right hand circular polarization (RHCP) and left hand circular polarization (LHCP), (2) a multipath signals model including amplitude, phase, and polarization, and (3) a ground reflection model applying to circular polarization signals. Based on the model, two kinds of novel up-to-down (U/D) ratios are presented. The performance of the model is assessed against the impact of up-to-down ratio of antenna on phase errors.
\end{abstract}

\section{Introduction}

Positioning in GNSS is based on the range measurements from the satellite antenna to the phase center of receiver antenna. The measurements are performed by utilizing phase delay of a pseudorandom noise (PRN) code or/and phase of the signal carrier. The satellite signals coming from different directions experience different delays of the PRN code and carrier phases. This can be interpreted as the effect of the variation of phase center [1].

For high-precision GNSS applications, the biases introduced by the phase center into the code phase and carrier phase measurements should be known and compensated. The tolerable residual measurements errors are at $\mathrm{cm}$ level for code and $\mathrm{mm}$ level for carrier measurements [2].

In order to mitigate the impact of phase center variations on high-precision GNSS, a careful determination of antenna phase center offsets and variations is required. Various methods for determination of the phase center have been proposed. Conventional phase center calibration methods include field calibration technique [3-5] and anechoic chamber calibration technique [6-8].
The field calibration is implemented in outfield using direct satellite signals. This requires suitable GNSS testing site and the same satellites signals for each measurement, so it has to be carried out for many days [9]. What is more, it is generally difficult to characterize the multipath using field data because the exact sources of the errors cannot be easily isolated. For anechoic chamber calibration technique, the calibration is implemented in anechoic chamber where the multipath signals are absent. When the antenna is used in outfield, the calibration values may be not useful because of the phase errors that arose by multipath. Multipath signals are the exact sources of the errors of phase center calibration.

In [10], a mathematical model of the corrupted signals composed of the direct line-of-sight signal and multipath signals of antenna is proposed. It allows evaluating the maximal phase measurement errors connected to each kind of parasite signal. In [11], carrier phase multipath parameters are identified and their influences on measurements are investigated through a theoretical analysis. A multipath simulation model is developed and described wherein the multipath parameters can be varied and their influences can be observed. For these models mentioned above, it 
considers that the antenna is modeled as point source, and the characteristics of polarization and radiation are ignored, while the receiving signal phase changes because of varying polarization and radiation.

A method of how to analyze the effect of multipath on geoquality GPS receivers based on amplitude pattern of a given antenna is described in [12]. In [13], to characterize the basic influence of multipath, a simple multiray signal model has been used. Based on the model, multipath phase rates for different environments were computed. Reference [14] studies the characteristics of reflected signal based on the $U / D$ ratio. In [15], a quantitative evaluation of multipath rejection capabilities of a GNSS antenna is introduced, and the technique is focused on the antenna pattern. For these models mentioned above, the multipath signal is assumed as pure RHCP or pure LHCP, while, in practical terms, the reflected signal includes not only RHCP part, but also LHCP part.

In this paper, we present a novel model of antenna receiving signal including direct and multipath signals; the model is further improved by adding antenna model and the characteristic of RHCP and LHCP. Based on the model, two kinds of $U / D$ ratios are presented. We also analyze the impact on phase errors that arose by multipath for different $U / D$ ratios. As an example, we use a choke antenna to assess the performance of the simulation results. We found that the multipath signal affects the phase evidently.

\section{Model of the Corrupted Signal}

2.1. Model of the Receiving Antenna. In general, the antenna polarization includes RHCP, LHCP, and linear polarization (LP). The LP can be converted to the linear combinations of RHCP and LHCP, so the universal antenna model can be represented as follows [16]:

$$
E_{\text {antenna }}(\theta, \varphi)=\left[\begin{array}{l}
\left|E_{L}(\theta, \varphi)\right| e^{j \theta_{L}(\theta, \varphi)} \\
\left|E_{R}(\theta, \varphi)\right| e^{j \theta_{R}(\theta, \varphi)}
\end{array}\right],
$$

where $\left|E_{L}(\theta, \varphi)\right|$ and $\theta_{L}(\theta, \varphi)$ are the amplitude and phase of the LHCP output in $(\theta, \varphi)$ direction; $\left|E_{R}(\theta, \varphi)\right|$ and $\theta_{R}(\theta, \varphi)$ are the amplitude and phase of the RHCP output in $(\theta, \varphi)$ direction; $\theta$ is the elevation angle; $\varphi$ is the azimuth angle.

In this paper, the horizontal plane is at $\theta=0^{\circ}$.

2.2. Model of Line-of-Sight Signal and Multipath Signal. The line-of-sight (LOS) signal can be written by the following formula:

$$
s_{d}(t)=A e^{j\left(\omega t+\theta_{0}\right)}, P_{d}
$$

with the following new parameters: $A$ is the signal amplitude; $\omega$ is the radian frequency; $\theta_{0}$ is the initial signal phase; $P_{d}$ is the polarization of the LOS signal.

The $P_{d}$ can be expressed by

$$
P_{d}=\left[\begin{array}{c}
\sin \gamma e^{j \varphi} \\
\cos \gamma e^{-j \varphi}
\end{array}\right],
$$

where $e^{j \varphi}, e^{-j \varphi}$ are the phase accumulated that arose by signal polarization, which is the typical character of circular polarization wave [17].

When $\gamma=45^{\circ}$ : LP. $\gamma=90^{\circ}$ : LHCP. $\gamma=0^{\circ}$ : RHCP, the satellite signal is RHCP in GNSS; in this paper, the LOS signal is RHCP. Consider the following:

$$
P_{d}=\left[\begin{array}{c}
0 \\
e^{-j \varphi}
\end{array}\right] .
$$

Multipath signal is introduced by reflection from the ground around the antenna. Compared to the LOS signal, the amplitude, phase, and polarization of the multipath signal change. Assuming that the reflected signal originates from a single point located on a ground, the multipath signal can be expressed by

$$
s_{m}(t)=\alpha A e^{j\left(\omega t+\theta_{0}+\theta_{m}\right)}, P_{m},
$$

where $\alpha$ is the attenuation factor which varies between 0 and $1, P_{m}$ is the polarization of the multipath signal, and $\theta_{m}$ is the phase change due to the reflection.

2.3. Ground Reflection Model. For a linear system, the Fresnel reflection factors are given by the following relationship [18]:

$$
\begin{aligned}
& r_{\perp}=\frac{\sin \theta-\sqrt{\varepsilon_{1}-j \delta_{1} / \omega \varepsilon_{0}} \sqrt{1-\left(1 / \varepsilon_{1}\right) \cos ^{2} \theta}}{\sin \theta+\sqrt{\varepsilon_{1}-j \delta_{1} / \omega \varepsilon_{0}} \sqrt{1-\left(1 / \varepsilon_{1}\right) \cos ^{2} \theta}}, \\
& r_{/ /}=\frac{\sqrt{\varepsilon_{1}-j \delta_{1} / \omega \varepsilon_{0}} \sin \theta-\sqrt{1-\left(1 / \varepsilon_{1}\right) \cos ^{2} \theta}}{\sqrt{\varepsilon_{1}-j \delta_{1} / \omega \varepsilon_{0}} \sin \theta+\sqrt{1-\left(1 / \varepsilon_{1}\right) \cos ^{2} \theta}},
\end{aligned}
$$

where $r_{\perp}$ is the Fresnel reflection factor for vertical polarization, $r_{/ /}$is the Fresnel reflection factor for horizontal polarization, $\theta$ is the incident angle, $\varepsilon_{0}$ is the free space dielectric, $\varepsilon_{1}$ is the relative dielectric constant, and $\sigma$ is the conductivity. So the reflected wave can be expressed by

$$
\left[\begin{array}{c}
E_{H \_R} \\
E_{V \_R}
\end{array}\right]=\left[\begin{array}{cc}
r_{/ /} & 0 \\
0 & r_{\perp}
\end{array}\right]\left[\begin{array}{c}
E_{H} \\
E_{V}
\end{array}\right],
$$

where $E_{H}, E_{V}$ are the complex phasor horizontal and vertical components of the incident signal and $E_{H_{-} R}, E_{V \_}$are the complex phasor horizontal and vertical components of the reflected wave.

The electric signal can be expressed using the sum of RHCP and LHCP components:

$$
E=\left[\begin{array}{l}
E_{L} \\
E_{R}
\end{array}\right],
$$

where $E_{L}, E_{R}$ are the complex phasor LHCP and RHCP components of the electric field.

Convert (8) to following formula [19]:

$$
\left[\begin{array}{c}
E_{H} \\
E_{V}
\end{array}\right]=\frac{1}{\sqrt{2}}\left|\begin{array}{cc}
1 & 1 \\
i & -i
\end{array}\right|\left[\begin{array}{c}
E_{L} \\
E_{R}
\end{array}\right] .
$$


The reflected signal can be written by the following formula:

$$
\begin{aligned}
{\left[\begin{array}{c}
E_{H_{-} R} \\
E_{V-R}
\end{array}\right] } & =\left[\begin{array}{cc}
r_{/ /} & 0 \\
0 & r_{\perp}
\end{array}\right]\left[\begin{array}{c}
E_{H} \\
E_{V}
\end{array}\right] \\
& =\frac{1}{\sqrt{2}}\left[\begin{array}{cc}
r_{/ /} & 0 \\
0 & r_{\perp}
\end{array}\right]\left|\begin{array}{cc}
1 & 1 \\
i & -i
\end{array}\right|\left[\begin{array}{c}
E_{L} \\
E_{R}
\end{array}\right],
\end{aligned}
$$

where $E_{H R}, E_{V R}$ are the complex phasor horizontal and vertical components of the reflected wave.

Convert (10) to the combinations of RHCP and LHCP; the reflected wave can be expressed by

$$
\begin{aligned}
{\left[\begin{array}{c}
E_{L \_R} \\
E_{R \_}
\end{array}\right] } & =\frac{1}{\sqrt{2}}\left|\begin{array}{cc}
1 & -i \\
1 & i
\end{array}\right|\left[\begin{array}{c}
E_{H_{-} R} \\
E_{V \perp}
\end{array}\right] \\
& =\frac{1}{2}\left[\begin{array}{ll}
r_{/ /}+r_{\perp} & r_{/ /}-r_{\perp} \\
r_{/ /}-r_{\perp} & r_{/ /}+r_{\perp}
\end{array}\right]\left[\begin{array}{c}
E_{L} \\
E_{R}
\end{array}\right],
\end{aligned}
$$

where $E_{L_{-}}, E_{R R}$ are the complex phasor LHCP and RHCP components of the reflected wave.

Adding the accumulated phase that arose by circular polarization to (11),

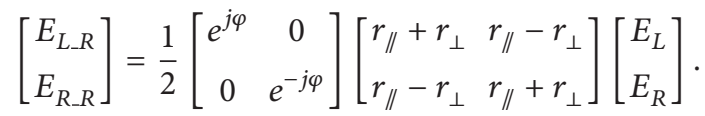

For a circular polarization system, the Fresnel reflection factor, $R$, can be written by the following formula:

$$
R=\frac{1}{2}\left[\begin{array}{cc}
\left(r_{/ /}+r_{\perp}\right) e^{j \varphi} & \left(r_{/ /}-r_{\perp}\right) e^{j \varphi} \\
\left(r_{/ /}-r_{\perp}\right) e^{-j \varphi} & \left(r_{/ /}+r_{\perp}\right) e^{-j \varphi}
\end{array}\right] .
$$

\section{Phase Errors Arose by Multipath Analysis}

3.1. Phase Errors Arose by Multipath [20]. There are two important multipath sources: signal reflected by a building and off the ground. In high-precision GNSS applications, the buildings are beneath the antenna; the signal reflected off the ground is the key multipath sources. In (5), $\theta_{m}=4 \pi h \sin \theta / \lambda$, where $\theta$ is the incident angle and $h$ is the height of the antenna above the reflected plane.

The received signal is given as the sum of the LOS and multipath signals:

$$
\begin{aligned}
s(t) & =s_{i d}(t)+s_{i m}(t) \\
& =s_{i d}(t)\left(1+\frac{e^{j \theta_{m}} E_{\text {antenna }}^{*}(-\theta, \varphi) \cdot R^{*} \cdot P_{d}}{E_{\text {antenna }}^{*}(\theta, \varphi) \cdot P_{d}}\right) \\
& =s_{i d}(t)\left(1+\alpha e^{j \phi}\right),
\end{aligned}
$$

where

$$
\begin{aligned}
& \phi=\angle \frac{e^{j \theta_{m}} E_{\text {antenna }}^{*}(-\theta, \varphi) \cdot R^{*} \cdot P_{d}}{E_{\text {antenna }}^{*}(\theta, \varphi) \cdot P_{d}}, \\
& \alpha=\left|\frac{e^{j \theta_{m}} E_{\text {antenna }}^{*}(-\theta, \varphi) \cdot R^{*} \cdot P_{d}}{E_{\text {antenna }}^{*}(\theta, \varphi) \cdot P_{d}}\right| .
\end{aligned}
$$

TABLE 1: Characteristics of dry and wet ground.

\begin{tabular}{lcc}
\hline Ground type & Relative dielectric constant & Conductivity \\
\hline Dry ground & 4 & 0.001 \\
Wet ground & 16 & 0.002 \\
\hline
\end{tabular}

So the phase errors that arose by multipath, $\Delta \phi$, can be obtained by

$$
\Delta \phi=\angle s(t)-\angle s_{i d}(t)=\operatorname{ar} \sin \left(\frac{\sin \phi}{1+\alpha^{2}+2 \alpha \cos \phi}\right) .
$$

The typically high-precision GNSS antenna has stable phase center. In this paper, we assume the phase center variation of antenna is zero and the $\varphi=0^{\circ}$; the conclusion is the same with other $\varphi$ values.

3.2. Errors Analysis in Outfield Environment. Equations (1), (4), and (15) can be arranged in the following formula:

$$
\begin{aligned}
& \phi=\angle \frac{\left|E_{L}(-\theta, \varphi)\right|\left(r_{\|}-r_{\perp}\right)+\left|E_{R}(-\theta, \varphi)\right|\left(r_{/ /}+r_{\perp}\right)}{2\left|E_{R}(\theta, \varphi)\right|}, \\
& \alpha=\left|\frac{\left|E_{L}(-\theta, \varphi)\right|\left(r_{\|}-r_{\perp}\right)+\left|E_{R}(-\theta, \varphi)\right|\left(r_{\|}+r_{\perp}\right)}{2\left|E_{R}(\theta, \varphi)\right|}\right| .
\end{aligned}
$$

As (17) predicts, we will see that the two major factors affecting the phase errors are the relative amplitude between the up RHCP and down RHCP and the relative amplitude between the up RHCP and down LHCP. Two formulas for $U / D$ ratio are defined as

$$
\begin{aligned}
& \frac{U}{D_{1}}(\theta, \varphi)=\frac{\left|E_{R}(\theta, \varphi)\right|}{\left|E_{R}(-\theta, \varphi)\right|}, \\
& \frac{U}{D_{2}}(\theta, \varphi)=\frac{\left|E_{R}(\theta, \varphi)\right|}{\left|E_{L}(-\theta, \varphi)\right|} .
\end{aligned}
$$

By rearranging (17), we can get the following relationship:

$$
\begin{aligned}
& \phi=\angle\left(\frac{\left(r_{/ /}+r_{\perp}\right)}{2 U / D_{1}}+\frac{\left(r_{/ /}-r_{\perp}\right)}{2 U / D_{2}}\right) e^{j \theta_{m}}, \\
& \alpha=\left|\left(\frac{\left(r_{/ /}+r_{\perp}\right)}{2 U / D_{1}}+\frac{\left(r_{/ /}-r_{\perp}\right)}{2 U / D_{2}}\right) e^{j \theta_{m}}\right| .
\end{aligned}
$$

In outfield environment, the wet ground and dry ground are two typical reflected materials, and the approximate values for dielectric constant and conductivity are given in Table 1 [21].

In our simulation, we choose the frequency GPS L1 $f=$ $1575.42 \mathrm{MHz}(\omega=2 \pi f)$, and the height of the antenna $h=$ $2 \mathrm{~m}$.

3.2.1. Dry Ground. Figure 1 depicts the phase errors that arose by dry ground reflected multipath changing against $U / D_{1}$ and $U / D_{2}$ for several values of elevation $\left(10^{\circ}-80^{\circ}\right)$. 


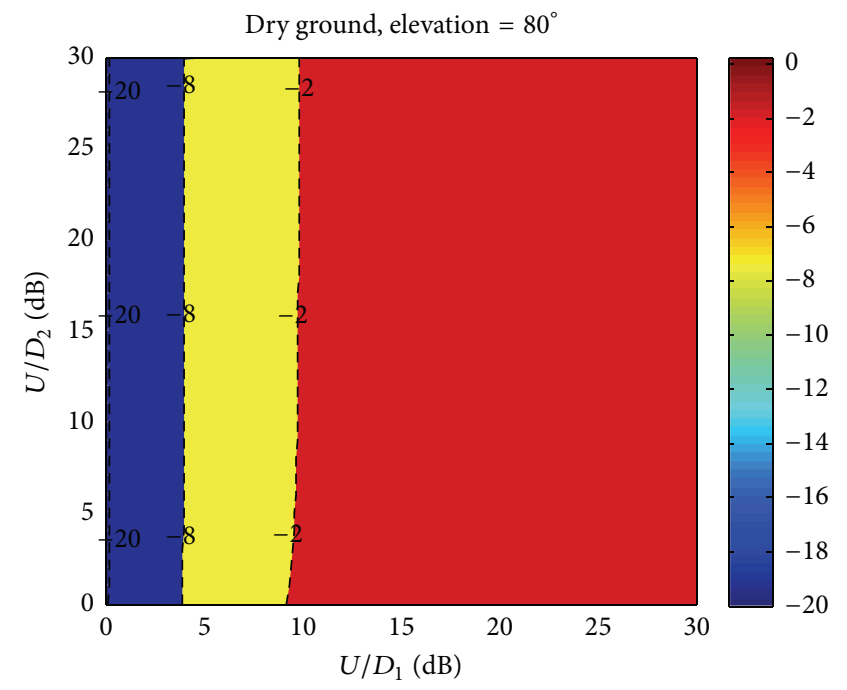

(a)

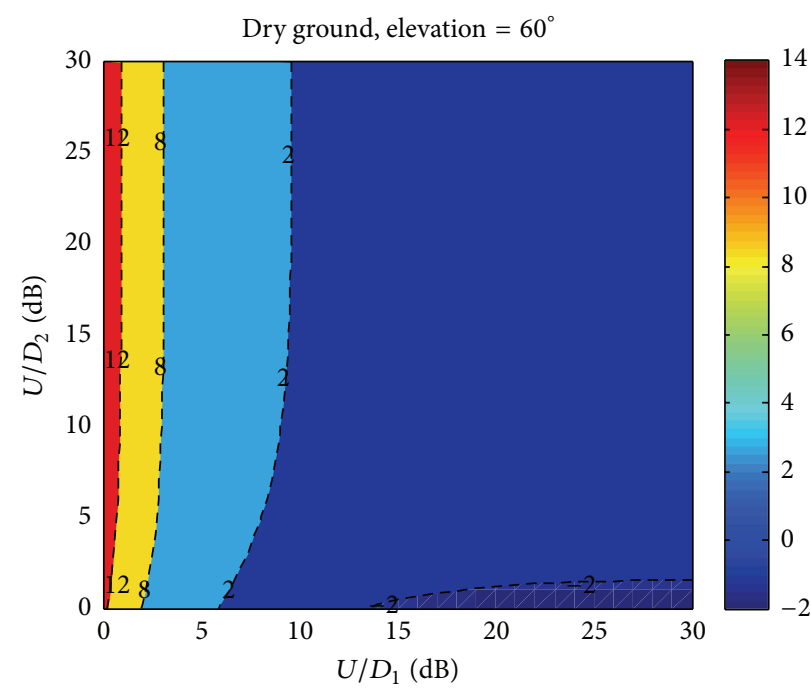

(c)

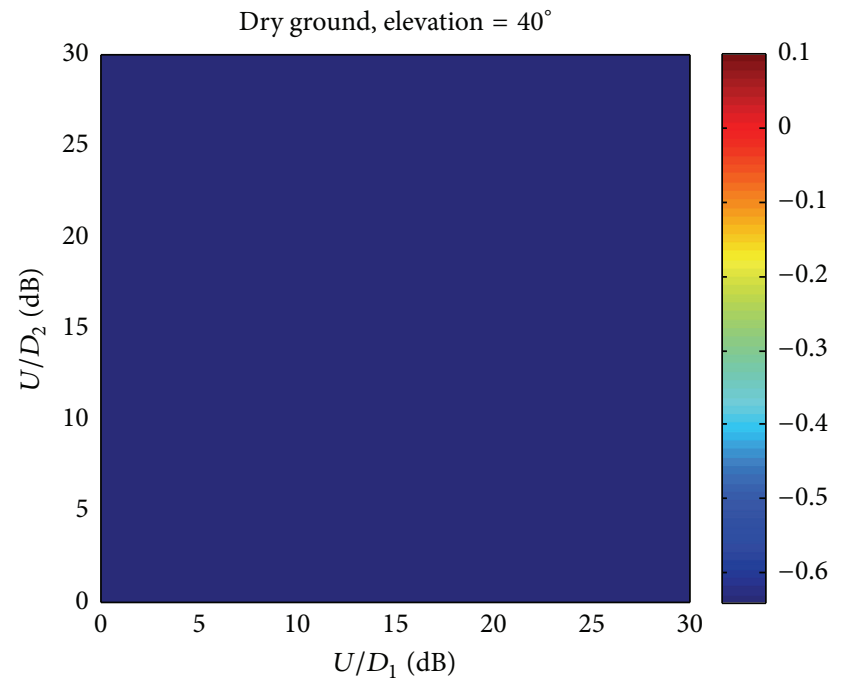

(e)

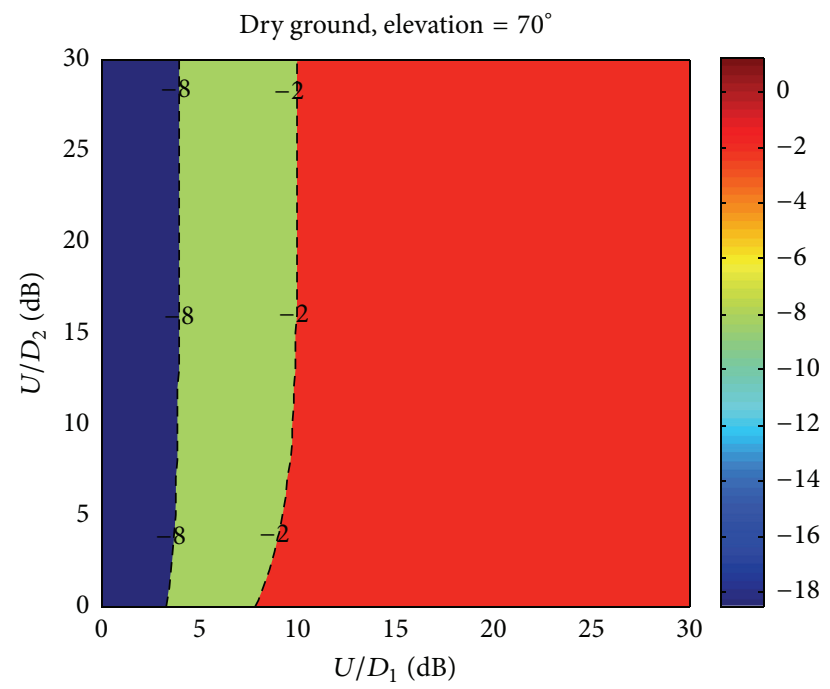

(b)

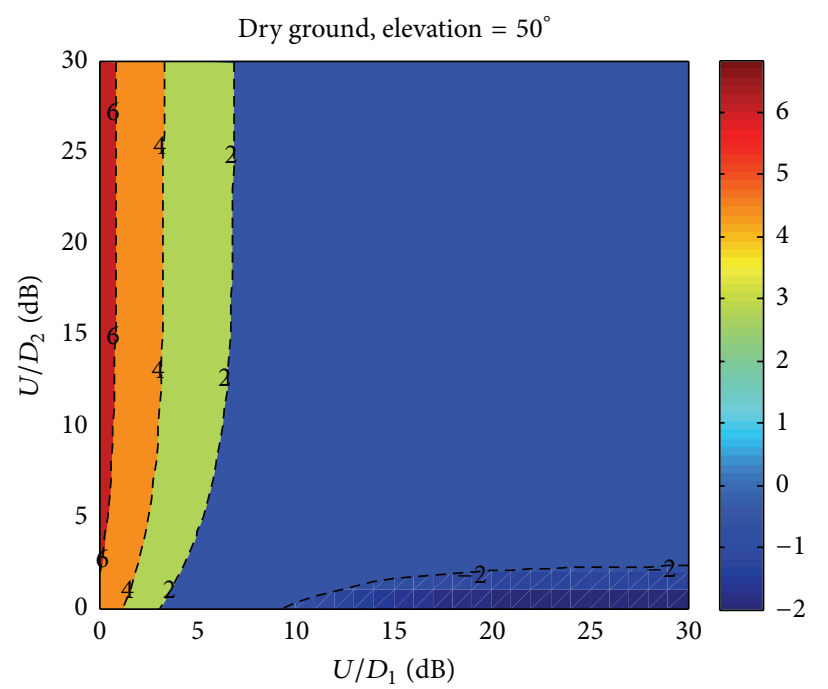

(d)

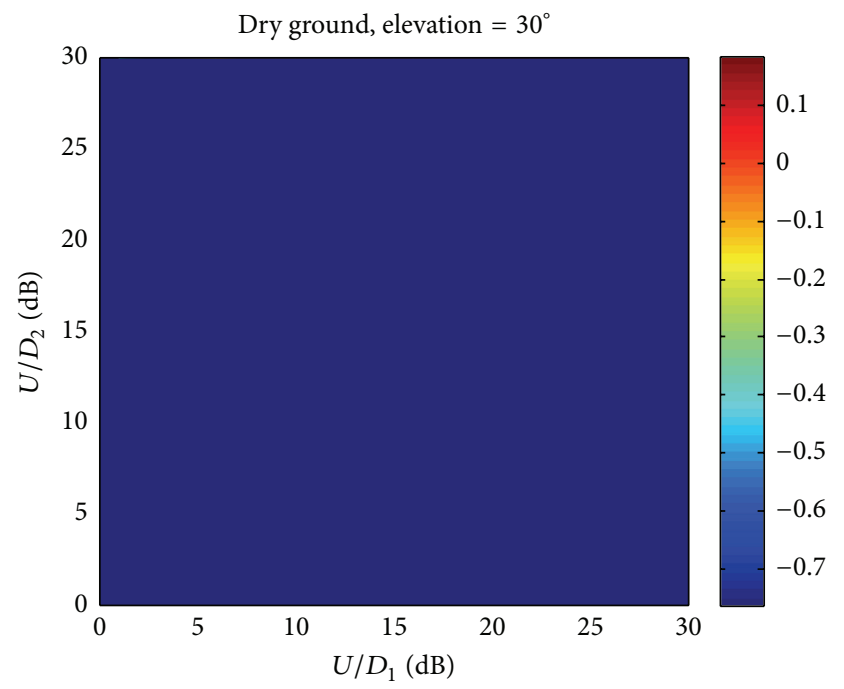

(f)

Figure 1: Continued. 


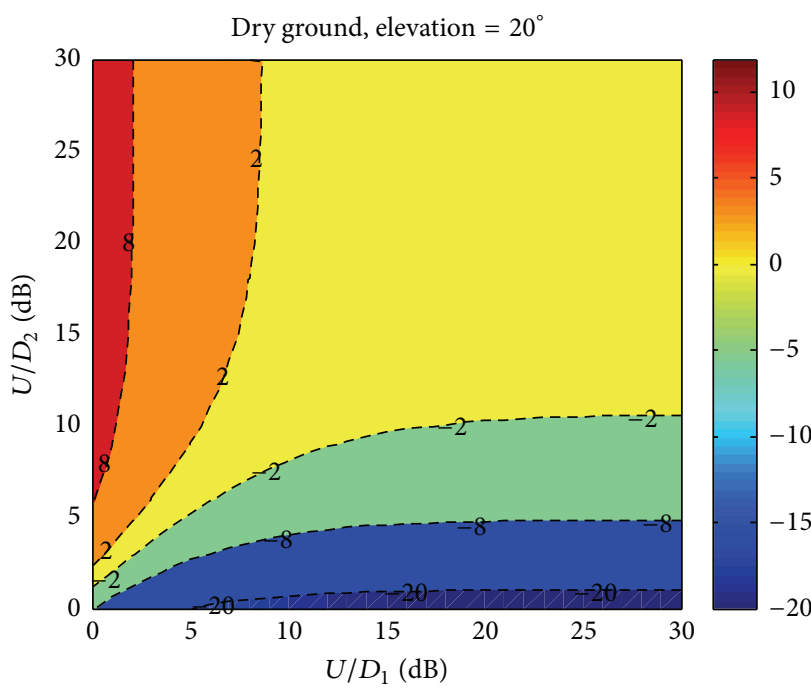

(g)

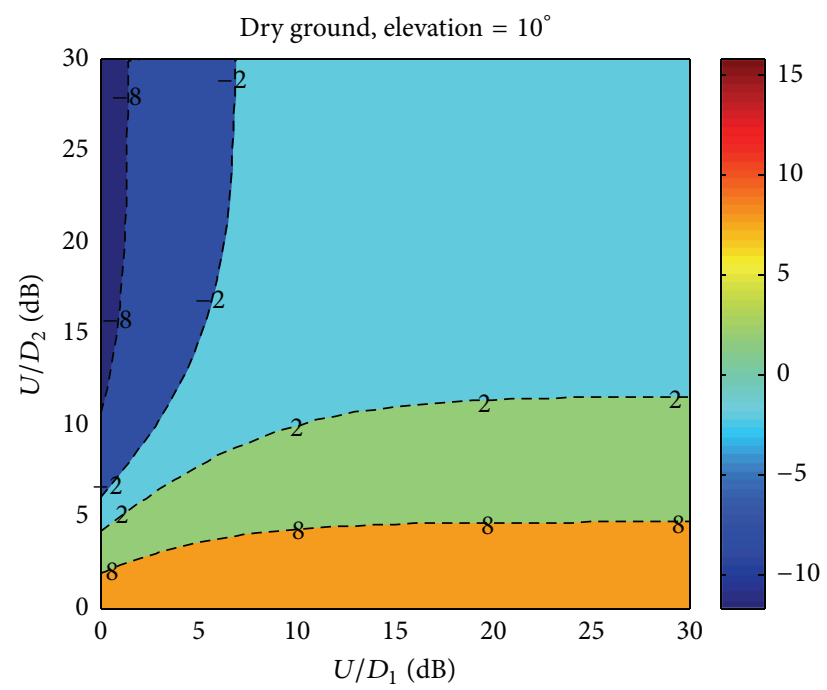

(h)

FiguRE 1: Effect of the multipath around dry ground on the carrier phase against $U / D_{1}$ and $U / D_{2}$ ratios for different elevation angles $(80,70$, $60,50,40,30,20$, and 10$)$.

As shown in Figure 1, we note that as $U / D_{1}$ and $U / D_{2}$ ratios increase, the phase errors decrease. For different elevations, the phase errors change differently against $U / D_{1}$ and $U / D_{2}$. When $U / D_{1}$ is higher than $10 \mathrm{~dB}$, the max phase error is below $2 \mathrm{~mm}(-2 \mathrm{~mm}<$ phase error $<2 \mathrm{~mm})$ at elevation $=80^{\circ}$ and $70^{\circ}$ (see Figures $1(\mathrm{a})$ and $1(\mathrm{~b})$ ). For elevation $=$ $40^{\circ}$ and $30^{\circ}$, the phase errors are always below $2 \mathrm{~mm}$ when $U / D_{1}>1 \mathrm{~dB}$; this is because the reflected wave is almost RHCP which is the same with the LOS wave. At elevation = $60^{\circ}, 50^{\circ}, 20^{\circ}$, and $10^{\circ}$, only when the $U / D_{1}$ and $U / D_{2}$ ratios are higher than a particular value, the phase errors are below $2 \mathrm{~mm}$, while the values are higher at $20^{\circ}$ and $10^{\circ}$ than those at $60^{\circ}$ and $50^{\circ}$.

3.2.2. Wet Ground. Figure 2 depicts the phase errors that arose by wet ground reflected multipath changing against $U / D_{1}$ and $U / D_{2}$ for several values of elevation $\left(10^{\circ}-80^{\circ}\right)$.

As shown in Figure 2, we note that the conclusion is similar to that at dry ground, while the reflected wave by wet ground is stronger than dry ground. In order to reduce the phase errors that arose by reflected multipath, higher $U / D_{1}$ and $U / D_{2}$ are demanded than dry ground.

3.2.3. Summary. When the phase center variations tolerance is $2 \mathrm{~mm}$ (typically), in order to mitigate the impact of phase center variations on high-precision GNSS by using calibration values in outfield environment where typical reflected materials are the wet ground or dry grounds, the different $U / D_{1}$ and $U / D_{2}$ ratios are demanded which can be obtained from Figures 1 and 2, as in Table 2.

\section{Examples}

In order to assess the performance of the simulation results, a choke antenna is analyzed. Figure 3 shows the RH and LHCP amplitude pattern of the antenna.
TABLE 2: The different $U / D_{1}$ and $U / D_{2}$ ratios demand for phase errors below $2 \mathrm{~mm}$ (approximate).

\begin{tabular}{lcccc}
\hline \multirow{2}{*}{ Elevation } & \multicolumn{2}{c}{ Dry ground } & \multicolumn{2}{c}{ Wet ground } \\
& $U / D_{1}$ & $U / D_{2}$ & $U / D_{1}$ & $U / D_{2}$ \\
\hline 80 & $>10 \mathrm{~dB}$ & - & $>13 \mathrm{~dB}$ & - \\
70 & $>10 \mathrm{~dB}$ & - & $>13 \mathrm{~dB}$ & - \\
60 & $>10 \mathrm{~dB}$ & $>2 \mathrm{~dB}$ & $>13 \mathrm{~dB}$ & $>2 \mathrm{~dB}$ \\
50 & $>7 \mathrm{~dB}$ & $>2 \mathrm{~dB}$ & $>10 \mathrm{~dB}$ & $>2 \mathrm{~dB}$ \\
40 & - & - & $>2 \mathrm{~dB}$ & - \\
30 & - & - & $>2 \mathrm{~dB}$ & - \\
20 & $>8 \mathrm{~dB}$ & $>8 \mathrm{~dB}$ & $>10 \mathrm{~dB}$ & $>9 \mathrm{~dB}$ \\
10 & $>8 \mathrm{~dB}$ & $>10 \mathrm{~dB}$ & $>9 \mathrm{~dB}$ & $>9 \mathrm{~dB}$ \\
\hline
\end{tabular}

Figure 4 shows the $U / D_{1}$ and $U / D_{2}$ of the antenna; as presented in Figure $4, U / D_{2}$ of the antenna is higher than $18 \mathrm{~dB}$ above horizontal, while $U / D_{1}$ is less than $12 \mathrm{~dB}$ at lowelevation angles (elevation $<10^{\circ}$ ). Figure 5 shows the effect of the multipath on the carrier phase against elevation angle for choke antenna. We note that the phase error is $5.28 \mathrm{~mm}$ for wet ground reflected and $6.01 \mathrm{~mm}$ for wet ground reflected at elevation $=10^{\circ}$, which is according to Figures $1(\mathrm{~g})$ and $2(\mathrm{~g})$. At high-elevation angles (elevation $>15^{\circ}$ ), the phase errors are all below $2 \mathrm{~mm}$.

When the phase center variations tolerance is $2 \mathrm{~mm}$, in order to mitigate the impact of phase center variations on high-precision GNSS application by using calibration values, the elevation cutoff angle will be set at $15^{\circ}$ or higher.

\section{Conclusions}

In this paper, we present a novel model of antenna receiving signal including direct and multipath signals; the model is 


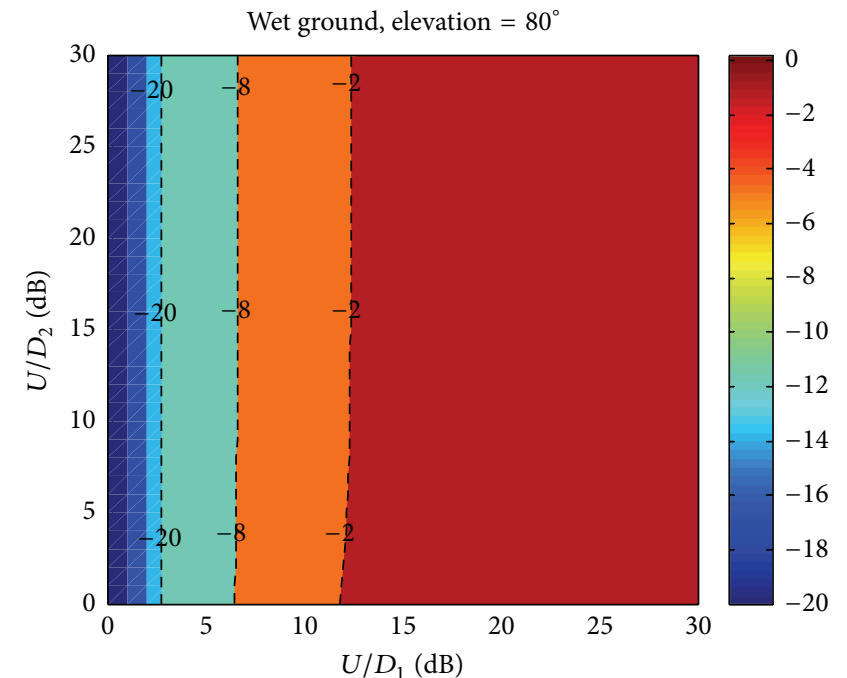

(a)

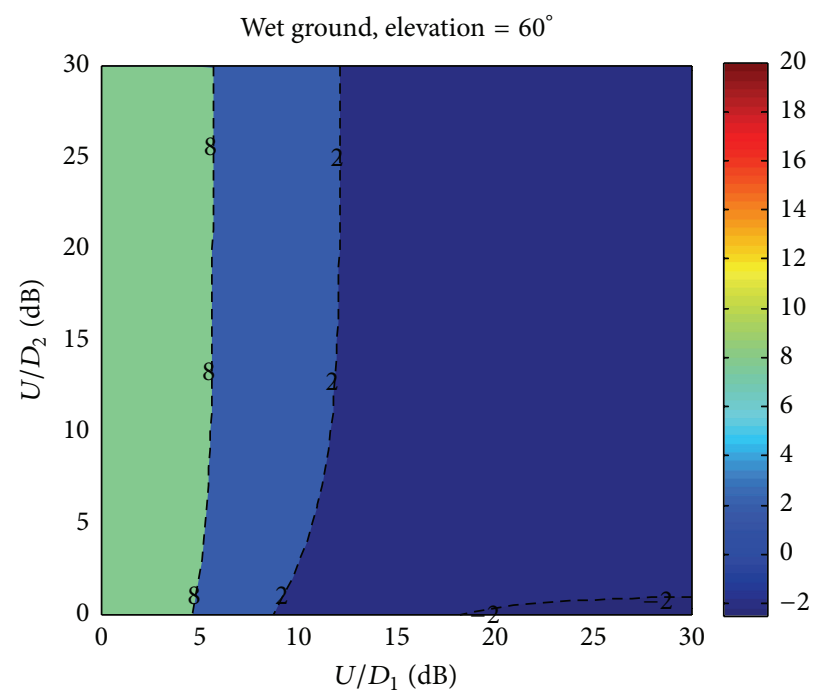

(c)

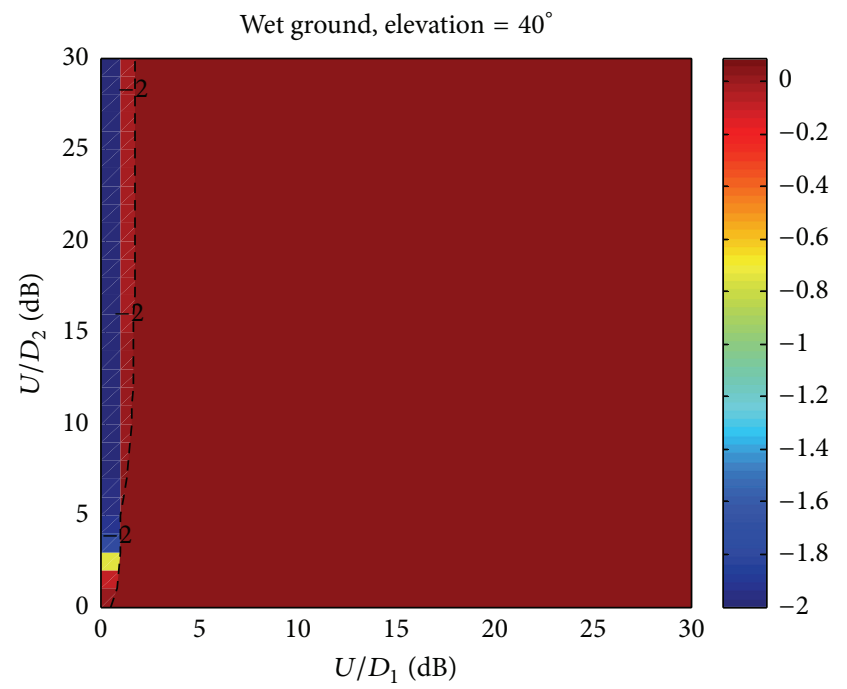

(e)

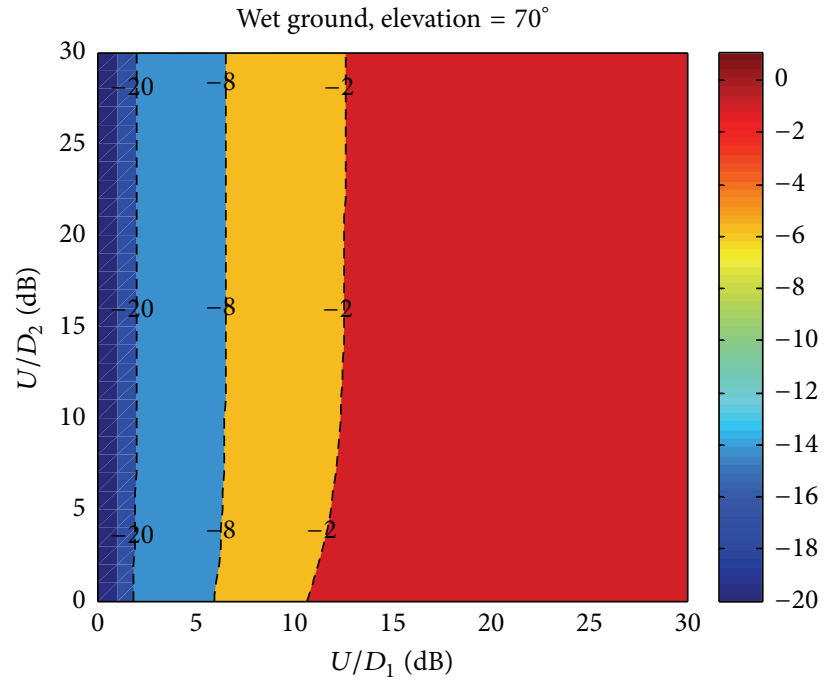

(b)

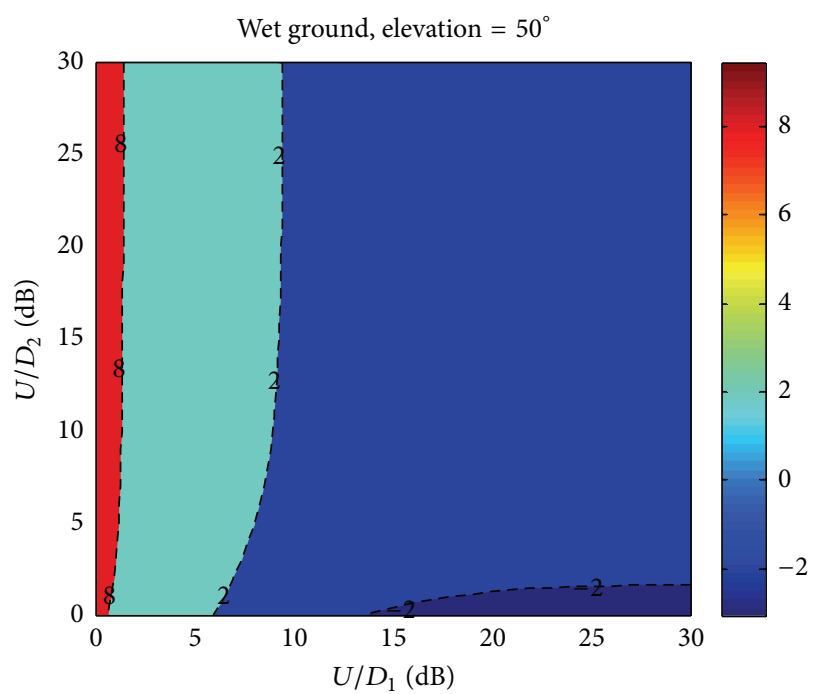

(d)

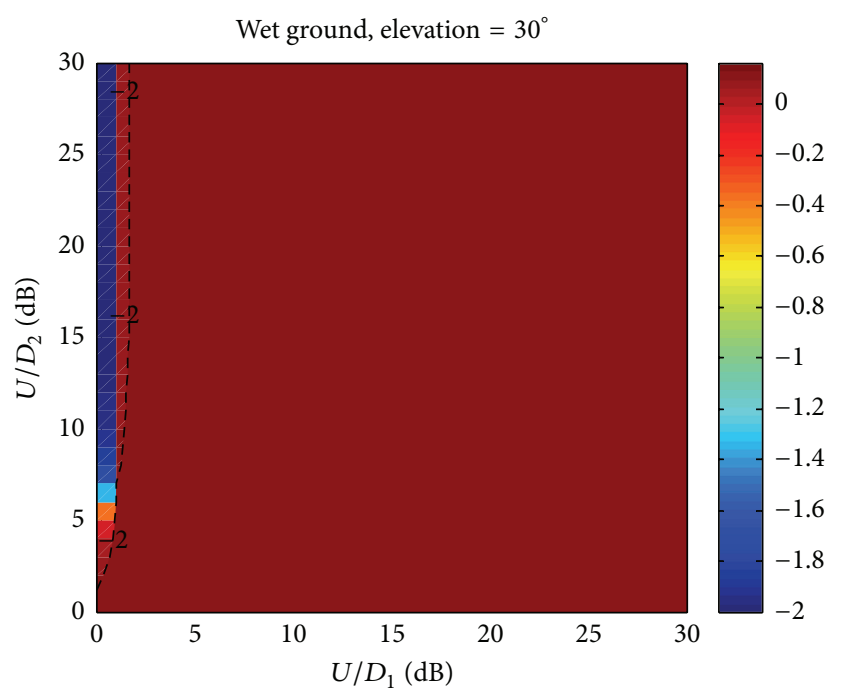

(f)

Figure 2: Continued. 


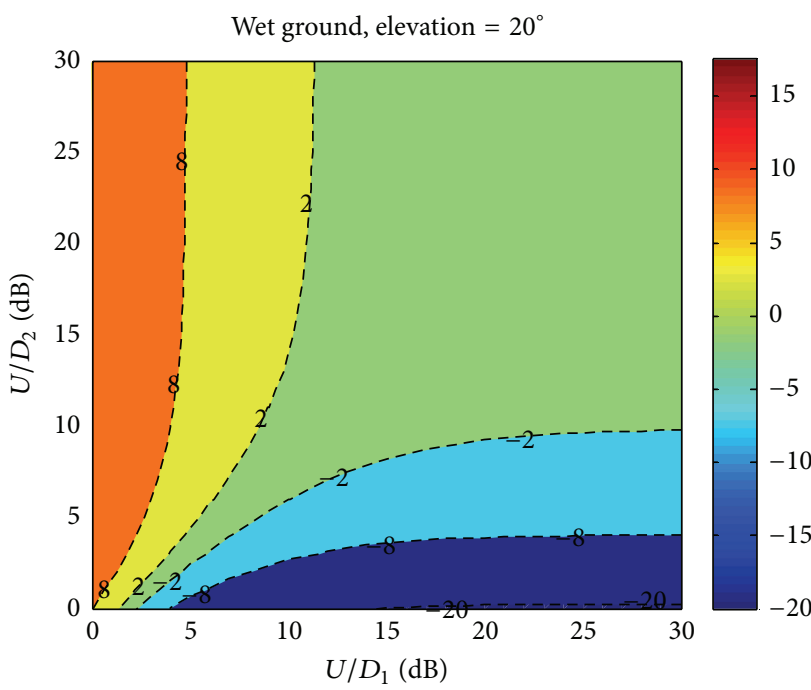

(g)

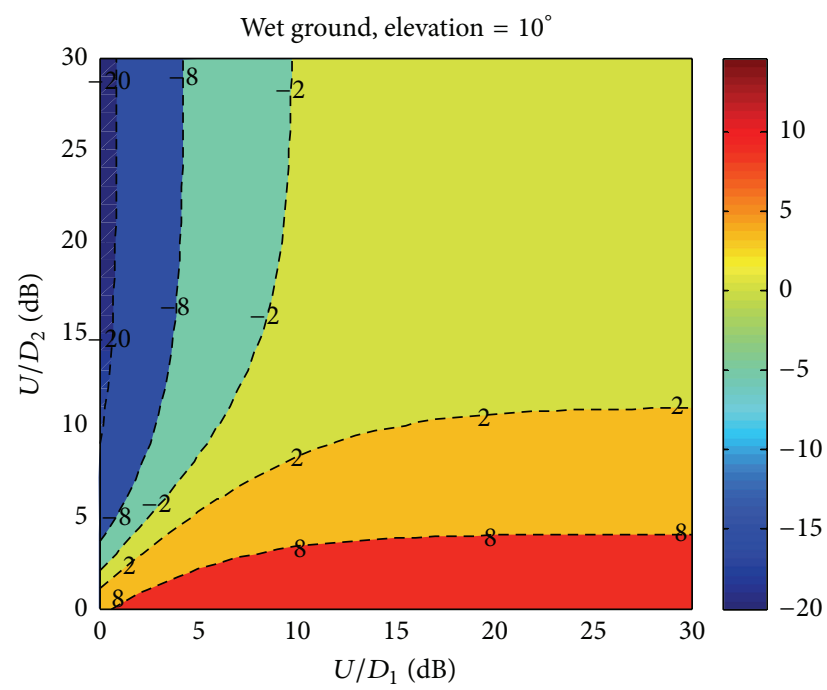

(h)

FIGURE 2: Effect of the multipath around wet ground on the carrier phase against $U / D_{1}$ and $U / D_{2}$ ratios for different elevation angles (80, $70,60,50,40,30,20$, and 10 ).

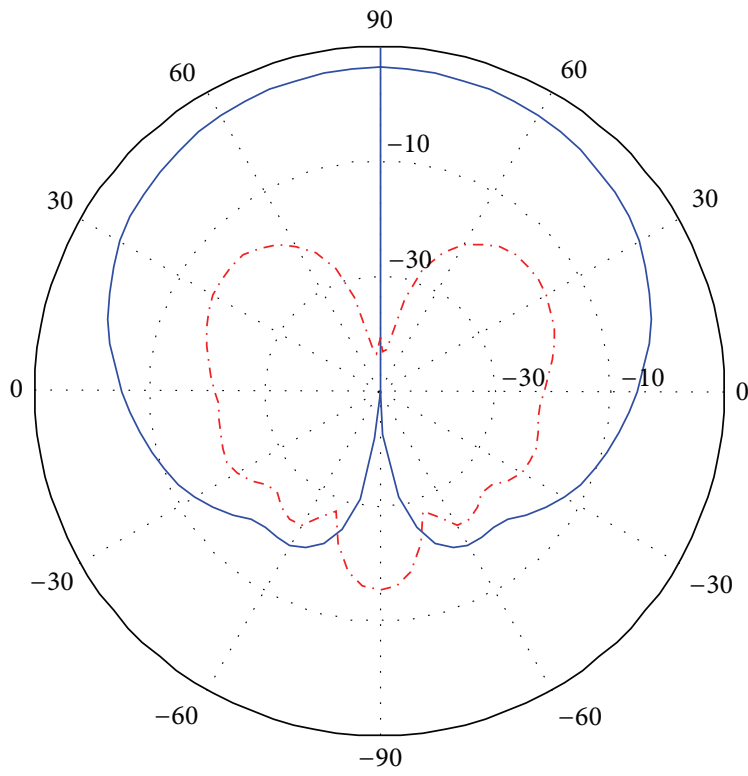

- RHCP $(\mathrm{dBi})$

-. L LHCP $(\mathrm{dBi})$

FIgURE 3: The RH and LHCP amplitude patterns for the choke antenna at $\mathrm{L} 1(\mathrm{phi}=0)$.

further improved by adding antenna model and the characteristic of RHCP and LHCP. We have applied the new antenna and multipath model to illustrate the phase errors that arose by multipath against $U / D_{1}$ and $U / D_{2}$; it was shown that the multipath signal affects the phase evidently; whichever of $U / D_{1}$ and $U / D_{2}$ ratio is below $15 \mathrm{~dB}$, the phase errors are

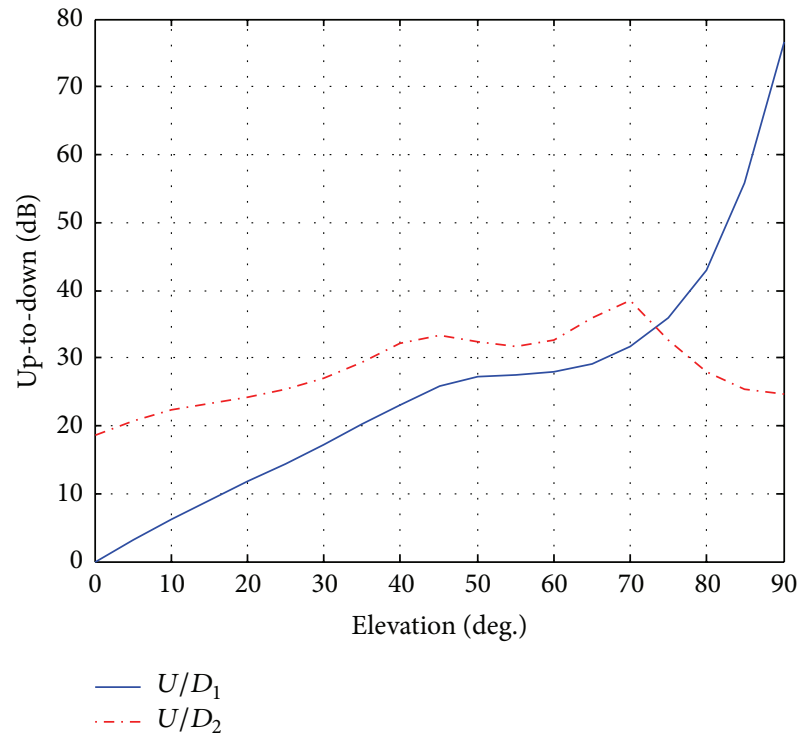

FIgURE 4: The $U / D$ ratios in $\mathrm{dB}$ for choke antenna.

higher than $2 \mathrm{~mm}$ that exceeds the phase center variations tolerance. In high-precision GNSS application, before using phase center calibration values, the following three steps are necessary:

(1) Obtain the RHCP and LHCP amplitude patterns.

(2) Calculate the $U / D_{1}$ and $U / D_{2}$ ratio of the antenna.

(3) Set the elevation cutoff based on the $U / D_{1}$ and $U / D_{2}$ ratio (in Table 2). 


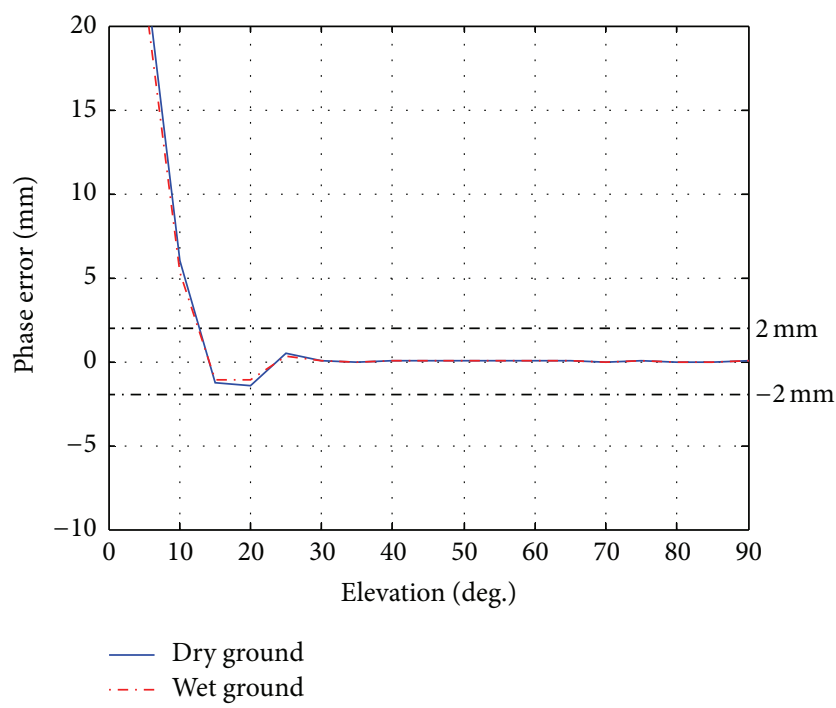

FIGURE 5: The effect of the multipath on the carrier phase against elevation angle for choke antenna.

In order to expand the coverage of antenna applied in high-precision GNSS application, we must heighten the $U / D_{1}$ and $U / D_{2}$ ratio at low-elevation angle.

\section{Conflict of Interests}

The authors declare that there is no conflict of interests regarding the publication of this paper.

\section{References}

[1] W. Kunysz, "Antenna phase center effects and measurements in GNSS ranging applications," in Proceedings of the 14th International Symposium on Antenna Technology and Applied Electromagnetics and the American Electromagnetics Conference (ANTEM/AMEREM '10), pp. 1-4, Ottawa, Canada, July 2010.

[2] L. Wanninger and M. May, "Carrier-phase multipath calibration of GPS reference stations," Navigation, vol. 48, no. 2, pp. 113-124, 2001.

[3] A. Kumar, A. D. Sarma, E. Ansari, and K. Yedukondalu, "Improved phase center estimation for GNSS patch antenna," IEEE Transactions on Antennas and Propagation, vol. 61, no. 4, pp. 1909-1915, 2013.

[4] O. Montenbruck, M. Garcia-Fernandez, Y. Yoon, S. Schön, and A. Jäggi, "Antenna phase center calibration for precise positioning of LEO satellites," GPS Solutions, vol. 13, no. 1, pp. 23-34, 2009.

[5] G. Wübbena, M. Schmitz, F. Menge, G. Seeber, and C. Völksen, "A new approach for field calibration of absolute GPS antenna phase center variations," Navigation, vol. 44, no. 2, pp. 247-255, 1997.

[6] J. R. Costa, E. B. Lima, and C. A. Fernandes, "Antenna phase center determination from amplitude measurements using a focusing lens," in Proceedings of the IEEE Antennas and Propagation Society International Symposium (APSURSI '10), pp. 1-4, IEEE, Toronto, Canada, July 2010.

[7] S. Perna, C. Esposito, A. Pauciullo, P. Romano, and A. Gifuni, "A fast approach for antenna phase center evaluation," in
Proceedings of the IEEE International Geoscience and Remote Sensing Symposium (IGARSS '14), pp. 1093-1096, IEEE, Québec, Canada, July 2014.

[8] Z. Y. Hu, Z. Li, O. Gang, and Z. Bin, "Research on antenna phase center anechoic chamber calibration method," in Proceedings of the International Conference on Microwave and Millimeter Wave Technology (ICMMT '10), pp. 1522-1524, IEEE, Chengdu, China, May 2010.

[9] A. Boussaad, S. Rock, and G. Alain, "Calibration antenna phase centers," GPS World, vol. 2, pp. 49-53, 2005.

[10] J. Godet, J. Dantepal, M. Grondin et al., "Multipath error analysis and calibration for GPS differential phase," in Proceedings of the Institute of Navigation National Technical Meeting (NTM '08), pp. 521-530, San Diego, Calif, USA, January 2008.

[11] J. Ray and M. Cannon, "Characterization of GPS carrier phase multipath," in Proceedings of the Institute of Navigation National Technical Meeting (NTM '08), pp. 343-352, San Diego, Calif, USA, January 2008.

[12] C. C. Chew, E. E. Small, K. M. Larson, and V. U. Zavorotny, "Effects of near-surface soil moisture on GPS SNR data: development of a retrieval algorithm for soil moisture," IEEE Transactions on Geoscience and Remote Sensing, vol. 52, no. 1, pp. 537-543, 2014.

[13] M. Irsigler, "Characterization of multipath phase rates in different multipath environments," GPS Solutions, vol. 14, no. 4, pp. 305-317, 2010.

[14] D. N. Aloi and M. S. Sharawi, "High fidelity antenna model validation results of a GNSS multipath limiting antenna," IEEE Transactions on Aerospace and Electronic Systems, vol. 47, no. 1, pp. 3-14, 2011.

[15] L. Boccia, G. Amendola, S. Gao, and C.-C. Chen, "Quantitative evaluation of multipath rejection capabilities of GNSS antennas," GPS Solutions, vol. 18, no. 2, pp. 199-208, 2014.

[16] R. C. Jones, "A new calculus for the treatment of optical systems: I. description and discussion of the calculus," Journal of the Optical Society of America, vol. 31, no. 7, pp. 488-493, 1941.

[17] F. Van Graas, C. Bartone, and T. Arthur, "GPS antenna phase and group delay corrections," in Proceedings of the Institute of Navigation National Technical Meeting (NTM), pp. 399-408, San Diego, Calif, USA, January 2004.

[18] I. de Arriba-Ruiz, F. Pérez-Martínez, and J. Maria MunozFerreras, "Time-reversal-based multipath mitigation technique for ISAR images," IEEE Transactions on Geoscience and Remote Sensing, vol. 51, no. 5, pp. 3119-3138, 2013.

[19] V. U. Zavorotny, K. M. Larson, J. J. Braun, E. E. Small, E. D. Gutmann, and A. L. Bilich, "A physical model for GPS multipath caused by land reflections: toward bare soil moisture retrievals," IEEE Journal of Selected Topics in Applied Earth Observations and Remote Sensing, vol. 3, no. 1, pp. 100-110, 2010.

[20] X. Wan and X. Zhan, "The research of multipath and linear error for pseudolites applications," Applied Mechanics and Materials, vol. 130-134, pp. 2890-2893, 2012.

[21] A. R. Lopez, "LAAS/GBAS ground reference antenna with enhanced mitigation of ground multipath," in Proceedings of the Institute of Navigation National Technical Meeting (NTM '08), pp. 389-393, San Diego, Calif, USA, January 2008. 

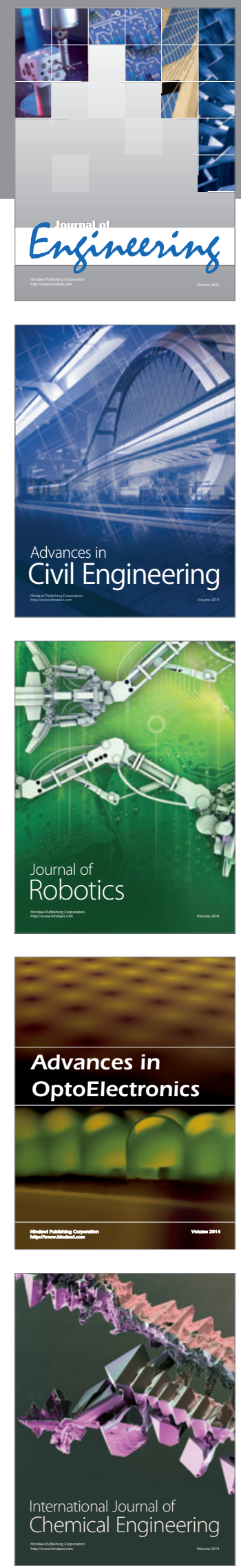

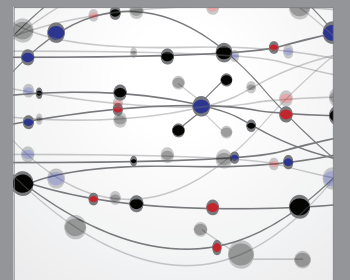

The Scientific World Journal
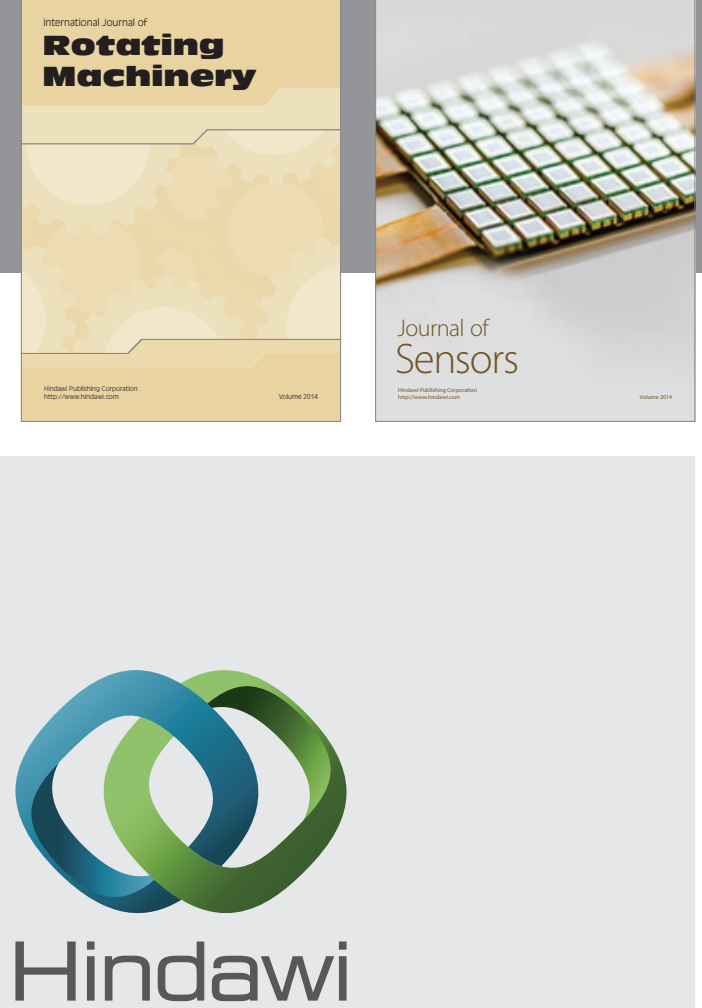

Submit your manuscripts at http://www.hindawi.com
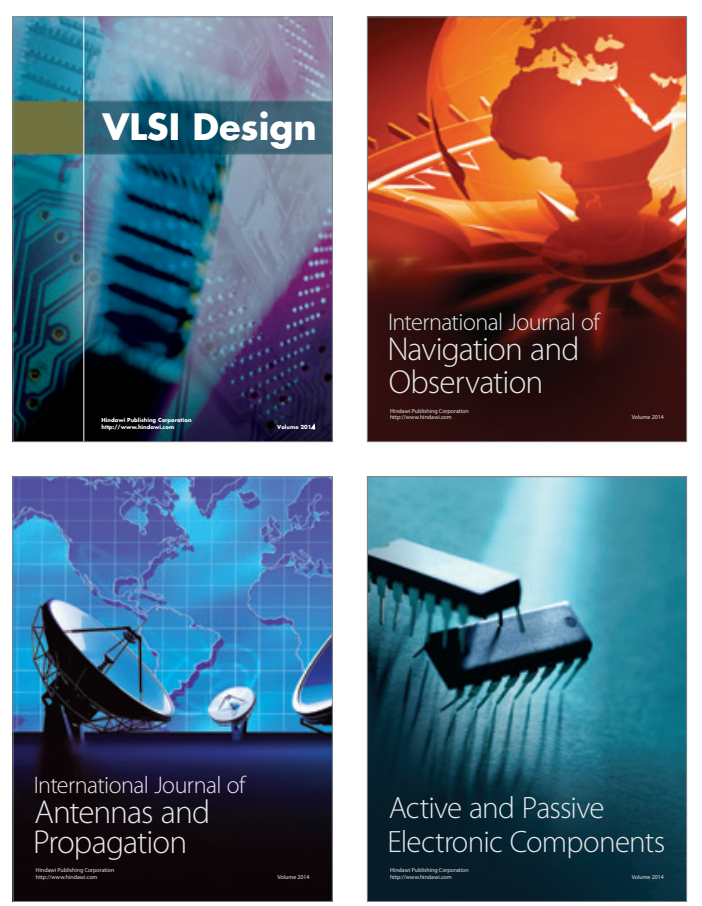
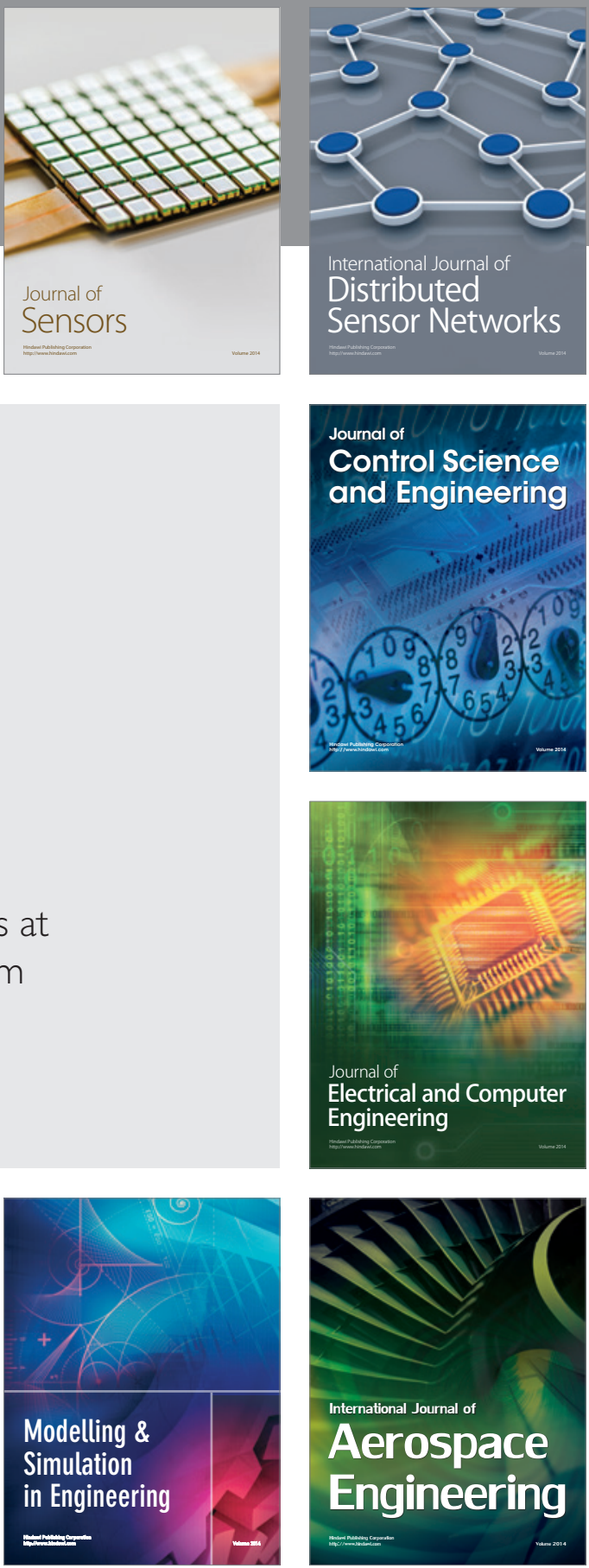

Journal of

Control Science

and Engineering
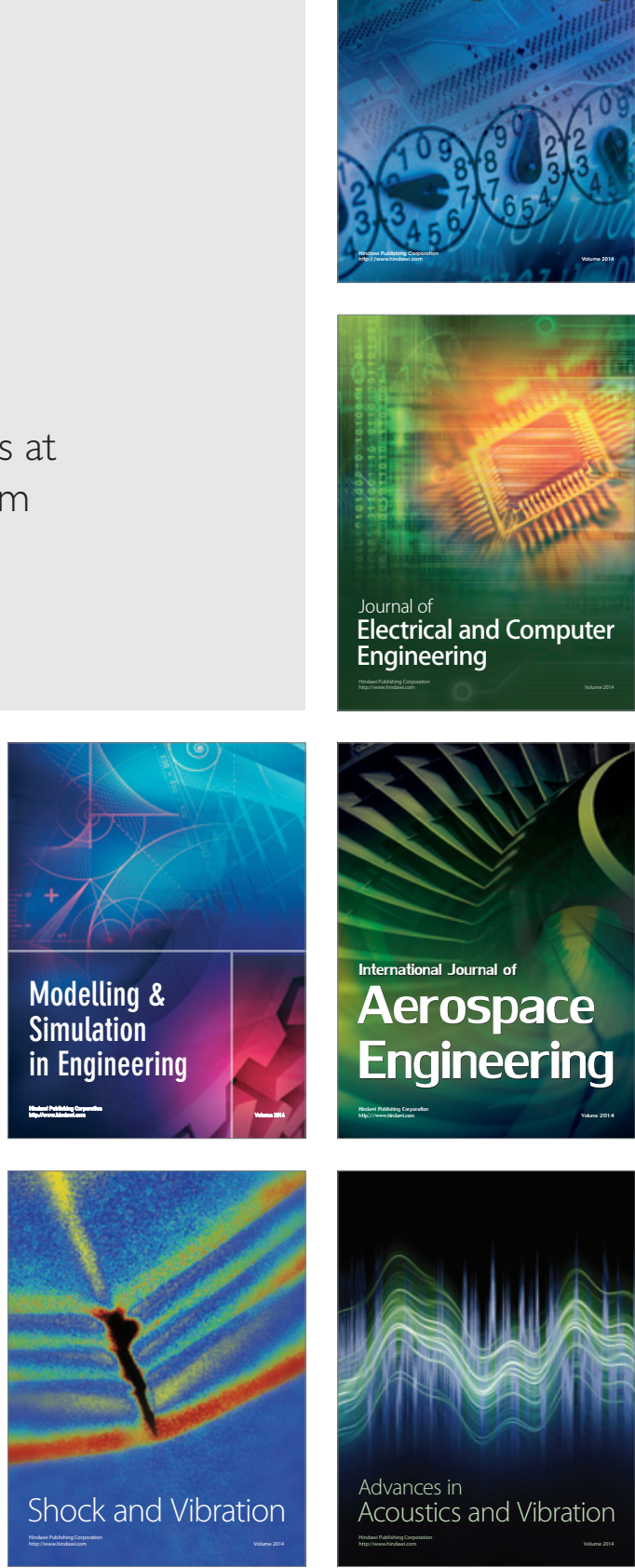